\title{
Magnetic resonance imaging evaluation after anorectal pull-through surgery for anorectal malformations: a comprehensive review
}

\author{
Saloni N. Desai ${ }^{1 A, E, F}$, Himanshu Choudhury ${ }^{1 B, C, D}$, Prashant Joshi' ${ }^{2 D, F}$, Sudheer Pargewar ${ }^{3 A, F}$ \\ 'Department of Radiology, Sir H. N. Reliance Foundation Hospital and Research Centre, Mumbai, India \\ ${ }^{2}$ Department of Paediatric Surgery, Sir H. N. Reliance Foundation Hospital and Research Centre, Mumbai, India \\ ${ }^{3}$ Department of Radiology, Gleneagles Global Hospital, Mumbai, India
}

\begin{abstract}
Anorectal malformations (ARM) include congenital anomalies of the distal anus and rectum with or without anomalies of the urogenital tract. Posterior sagittal anorectoplasty (PSARP) and minimally invasive laparoscopically assisted anorectal pull-through (LAARP) procedure are now mainly used to surgically treat ARMs. Magnetic resonance imaging (MRI) is the modality of choice for interval follow-up assessment of structural and functional outcome after these surgeries to assess future bowel continence.

Well-developed pelvic musculature has been found to be a reflector of better anal continence after ARM surgery. Thus, MRI plays an important role in evaluating the external sphincter complex, puborectalis, and levator ani muscles. Other parameters that need to be noted include the position of the neoanus, rectal diameter, anorectal angle, presence or absence of megarectum, and other ancillary anomalies in the spine.
\end{abstract}

Thus, MRI due to superior soft-tissue resolution is the modality of choice and indispensable for post-operative pelvic evaluation in children.

Key words: magnetic resonance imaging (MRI), anorectal malformations (ARM), PSARP, pelvis.

\section{Introduction}

Anorectal malformations (ARM), with a prevalence of $1: 5000$ live births, refer to the congenital anomalies of the distal anus and rectum often associated with anomalies of the urogenital tract. Various traditional and now minimally invasive surgeries aim to improve long-term faecal continence, normal bowel habits, and quality of life in these patients [1]. Posterior sagittal anorectoplasty (PSARP), described by de Vries and Pena in 1982, has been used widely in the surgical correction of ARMs. The procedure involves a midline sagittal incision from the sacrum to perineum, splitting the ileo and pubococcygeal portions of the levator, striated external sphincter muscle complex, pubococcygeus, and presumed puborectalis. The distal bowel is then mobilised and a neo-anus is reconstructed by suturing the terminal bowel wall to the striated muscles and mucosa to skin [2]. With the description of a minimally invasive laparoscopically assisted anorectal pull-through (LAARP) procedure by Georgeson et al. in 2000, this technique is now gaining momentum for management of intermediate and high ARMs. Its advantages include minimal surgical stress, better visualisation of rectal fistula and perineal structures, and accurate midline placement of pulled bowel within the levator ani and external sphincter complex musculature. Interval follow-up assessment of structural and functional outcome after these surgeries is essential to obtain information about future bowel function and for toilet training [3]. Functional scoring systems include subjective and clinical evaluation for bowel function. Magnetic resonance imaging (MRI), because of its superior soft tissue contrast,

Correspondence address:

Saloni N. Desai, Department of Radiology, Sir H. N. Reliance Foundation Hospital and Research Centre, Mumbai, India, e-mail: saludesai@gmail.com

Authors' contribution:

A Study design · B Data collection · C Statistical analysis · D Data interpretation · E Manuscript preparation · F Literature search · G Funds collection 
is a gold-standard imaging modality for evaluation of post-surgical pelvic floor changes.

\section{Magnetic resonance imaging technique}

MRI due to its multiplanar imaging and higher soft tissue contrast resolution is now regarded as an indispensable imaging modality for the evaluation of pelvic structures and musculature. With the advancement in technology, MRI is recommended on at least $1.5 \mathrm{~T}$ machines or higher. Wong et al., in their study on post-operative evaluation of children after laparoscopic anorectoplasty for imperforate anus, recommend MRI of the pelvis at least six months after surgery [4]. Pre-scanning preparation includes a soft liquid diet 12 hours prior to scanning and an enema or suppository on the morning of the scan. For better identification of the anus after pull-through, a catheter may be placed in the anus if needed. A body or pelvic phase array coil is used and an initial sagittal T2-weighted fast spinecho (FSE) sequence is performed to understand the axis of the anal canal. We use a sagittal T2 FSE sequence with a repetition time of 3200 to $3700 \mathrm{~ms}$ and an echo time of 90 to $115 \mathrm{~ms}$ with a matrix size of $448 \times 448$ to additionally cover the sacrum and lower lumbar vertebrae. Oblique axial T1- (repetition time of 400 to $600 \mathrm{~ms}$ and echo time of $20 \mathrm{~ms}$ ) and T2-weighted FSE sequences are obtained parallel to the pubococcygeal line [1]. Oblique coronal T1- and T2-weighted FSE sequences are performed perpendicular to the oblique axial images. A fat-suppressed, T2-weighted sequence in the axial or coronal plane may be added to evaluate fluid collections or inflammation in the surgical bed. In uncooperative and agitated patients, the MRI study can be performed under sedation.

\section{Magnetic resonance imaging assessment}

A semiquantitative MRI scoring system was proposed by Wong et al. to analyse the following four parameters and to standardise the reporting structure in these postoperative patients: (a) the striated muscle complex sphincter thickness symmetry/asymmetry was compared on either side; (b) the outline and regularity of the sphincter, whether smooth or irregular, to rule out perirectal fibrosis; (c) the position of the pull-through rectum whether in central or off-midline as regards to the pelvic floor; and (d) presence or absence of megarectum [4]. They analysed these structures at the level of ischial rami (I-plane) and at a level midway between the pubococcygeal line (PC plane) and I-plane. Another study conducted post repair of high anorectal malformations performed MRI on children at three years of age and assessed development of puborectalis, external sphincter muscles and levator hammock, symmetric passage of rectum or pulled intestine, anorectal angle, and rectal diameter [5]. The aforementioned muscle development was graded as 'good' if the development was comparable to normal subjects without any anorectal malformation, 'fair' if muscles were identified but not adequately developed and 'poor' in patients of barely identifiable or non-identifiable muscles. The external sphincter is evaluated on axial MRI, while the levator ani is assessed on coronal images. The anorectal angle was calculated on the mid-sagittal MRI image.

\section{Discussion}

PSARP and LAARP are now widely used surgical techniques for the management of ARMs; however, there are a lack of standardised criteria to judge long-term outcome after these procedures. The assessment of bowel function and anal continence is essential to determine prognosis and future outcome, and Kelly's clinical scoring is widely used as an appropriate technique for evaluating continence in post-operative patients. While the use of manometry for functional assessment of clinical continence and anal resting pressures has also been reported, no universal standard is in place and findings have been conflicting in different studies [6,7]. Previously, the role of CT scan in operated patients of ARM to identify the location of correctly placed pulled-through intestine was described by Taccone et al. [8]. State of the art MRI scanners with their higher soft tissue resolution and lack of radiation, especially essential in paediatric patients, is now one of the preferred modalities for perineal evaluation. Similarly, Vade et al. suggested the substitution of CT scan with MRI to look for anorectal sphincter after rectal pullthrough surgery [9].

Studies have shown that well-developed pelvic musculature is associated with better anal continence after ARM surgery, and hence MRI evaluation of the external sphincter complex and levator ani muscles is of paramount importance $[4,5,10]$ (Figures 1, 2, and 3). A study conducted by Wong et al. concluded that patients operated using the laparoscopic approach had better sphincter symmetry and lesser irregularity and perirectal fibrosis as compared to those who underwent PSARP [4]. Also, patients who have undergone a single-stage PSARP have been found to have better developed muscles as compared to those who have undergone traditional repair of high anorectal malformations [5]. Conflicting reports are available regarding the status and asymmetry of the pulled-through intestine, and Gangopadhyaya et al. in a study on 40 patients did not find any significant association between anal continence and asymmetry of the pulled-bowel. They also found a statistically significant correlation between anal continence and rectal diameter in operated patients, with a diameter range of 2.3 to $2.4 \mathrm{~mm}$ (mean $2.36 \mathrm{~mm}$ ) related with better continence as compared to rectal diameter range of 2.95 to $3.3 \mathrm{~mm}$ (mean $3.16 \mathrm{~mm}$ ) [5].

Patients with a lower obtuse anorectal angle have a better clinical outcome, and mean suggested values for good, fair, and poor anal continence are $113.72 \pm 7.4^{\circ}$, $132.9 \pm 10.7^{\circ}$, and $145.13 \pm 7.1^{\circ}$, respectively, and thus 

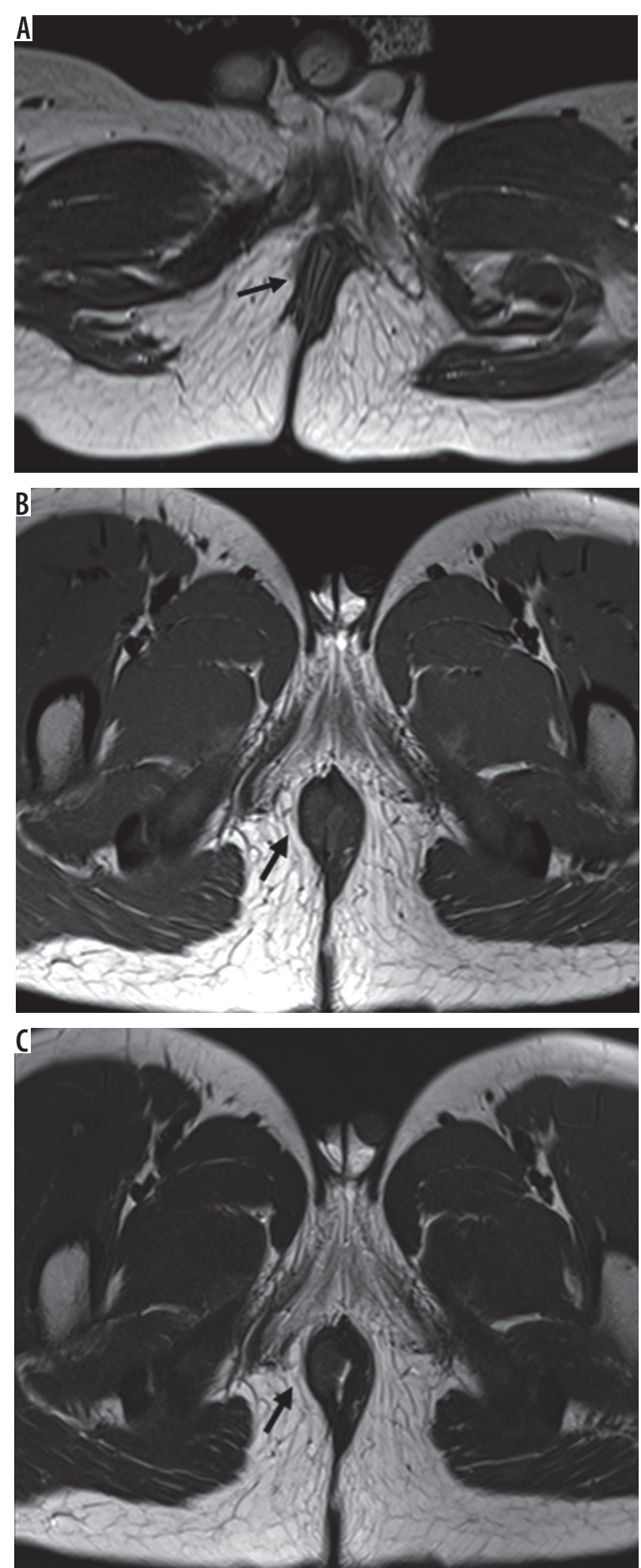

Figure 1. A) Axial magnetic resonance imaging (MRI) perineum in a normal child showing normal development, appearance, and symmetry of the external sphincter (arrow). B-C) Underwent posterior sagittal anorectoplasty for intermediate anorectal malformations at 4 months of age, complains of soiling. MRI done at 7 years of age, axial T1W (B), and axial T2W (C) image showing external sphincter asymmetry, poor development with right mucosal prolapse (arrows)

anorectal angle can be used as a predictor for post-surgical continence $[5,10,11]$ (Figure 4). Anorectal angulation in postoperative patients is indicative of adequate striat-

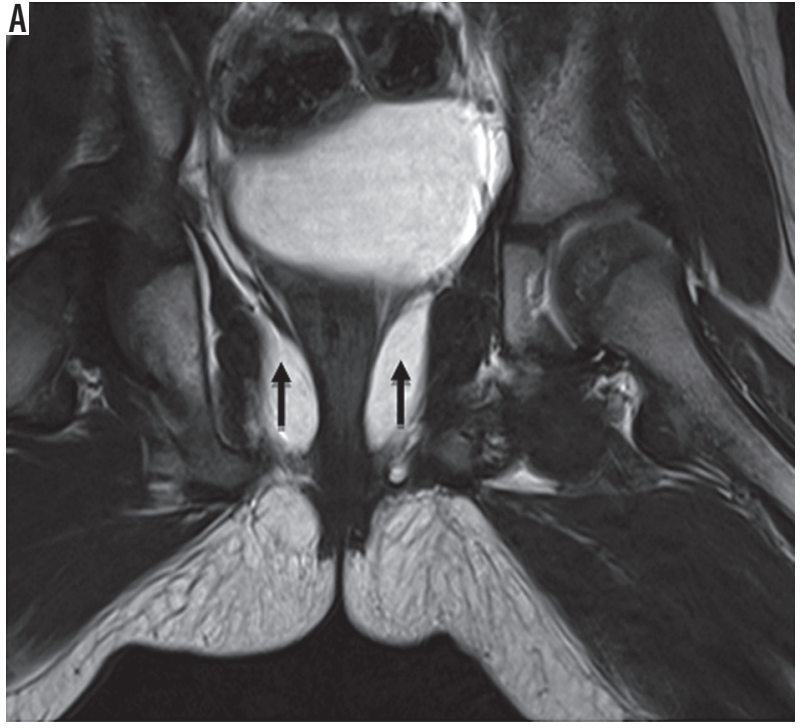

B

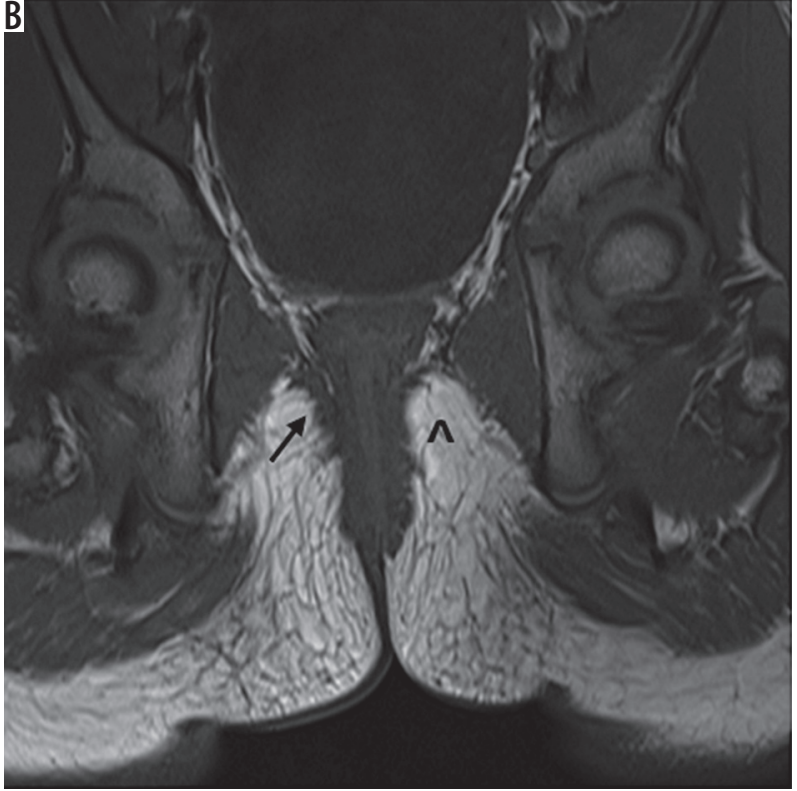

Figure 2. A) Coronal T2W magnetic resonance imaging (MRI) reveals normal appearance of the levator hammock (arrows). B) MRI of post posterior sagittal anorectoplasty child 1 year after surgery revealing asymmetry of levator hammock with fair development of the muscle on right side (arrow) and poor development on left side (arrowhead)

ed muscle complex contraction, and prior barium enema studies also report a good correlation between the degree of post-surgical anal continence and anorectal angulation $[12,13]$.

The presence of megarectum should be ruled out in patients suffering from intractable constipation after ARM surgery because it may be masquerade and represent faecal pseudo incontinence [14]. Additionally, MRI of the spine can be carried out in the same setting to rule out any vertebral column or spinal cord anomalies. Thus, a proposed MRI reporting format after pull-through surgery for ARMs is as described in Table 1.

Alternatively, the value of anal endosonography (AES) following the repair of anorectal malformations has been described by Jones et al., who compared AES and MRI in 


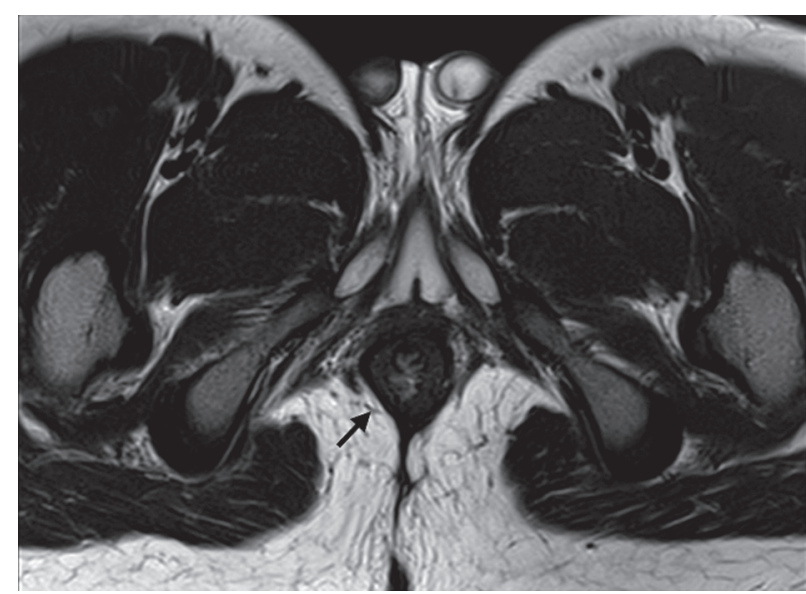

Figure 3. Post posterior sagittal anorectoplasty axial magnetic resonance imaging (seen at the level of ischial rami - I plane) of a patient 2 years after surgery showing well-developed symmetric external sphincter complex (arrow)

Table 1. Parameters to assess on magnetic resonance imaging after anorectal pull-through surgery for anorectal malformations

\begin{tabular}{|l|c|}
\hline $\begin{array}{l}\text { Degree of development of external } \\
\text { sphincter and levator ani muscles }\end{array}$ & Good/fair/poor \\
\hline $\begin{array}{l}\text { External sphincter muscle } \\
\text { thickness symmetry }\end{array}$ & Symmetric/asymmetric \\
\hline Outline of sphincter muscle & Smooth/irregular \\
\hline Position of pulled-through intestine & Centre/off-centre \\
\hline Rectal diameter & - \\
\hline Anorectal angle & - \\
\hline Megarectum & Absent/present \\
\hline Additional findings & Vertebral, spinal cord anomalies \\
\hline
\end{tabular}

14 patients following anorectoplasty. AES was performed under sedation one month and five years after surgery using a $10-\mathrm{MHz}$ transducer rotating through $360^{\circ}$ and evaluated the neo-anus and the sphincter. They concluded that AES had a better correlation with muscle stimulation and was a reliable alternative to MRI [15]. Ichijo et al. used both AES and MRI to assess the outcome of surgery for high/intermediate-type imperforate anus and measured the thickness of external sphincter and puborectalis post-surgery in the 3 and 9 o'clock positions. Because MRI can measure the thickness only on a particular image, they found MRI alone to be unreliable vis-à-vis AES [16].

\section{Conclusions}

MRI is an adequate modality in patients following pullthrough surgery for ARM because it aids in overall prognostication of anal continence and functional outcome. Accurate delineation of pelvic soft tissue anatomy and
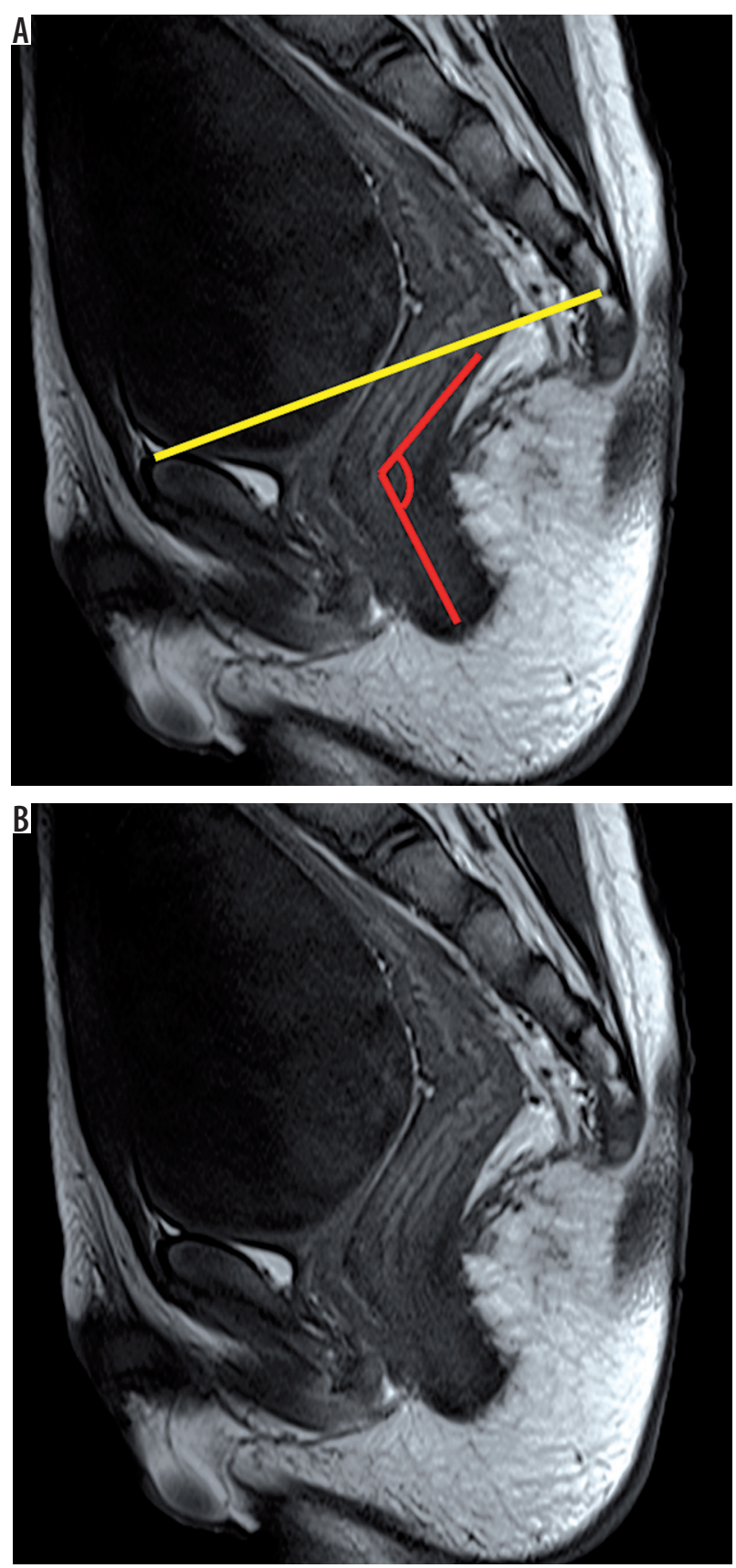

Figure 4. A) Depiction of pubococcygeal plane (yellow line) and anorectal angle (in red) on sagittal image. B) Post PSARP patient 1 year after surgery with good anal continence. Sagittal magnetic resonance imaging showed a lower obtuse anorectal angle $\left(110^{\circ}\right)$

the status of pelvic floor musculature is a significant factor in predicting long-term clinical progress and can also be used as an objective standard in subsequent follow-up examinations.

\section{Conflict of interest}

The authors declare that they have no conflict of interest. 


\section{References}

1. Alamo L, Meyrat BJ, Meuwly JY, et al. Anorectal Malformations: Finding the Pathway out of the Labyrinth. Radiographics 2013; 33: 491-512.

2. deVries PA, Peña A. Posterior sagittal anorectoplasty. J Pediatr Surg 1982; 17: 638-643.

3. Georgeson KE, Inge TH, Albanese CT. Laparoscopically assisted anorectal pull-through for high imperforate anus - a new technique. J Pediatr Surg 2000; 35: 927-930.

4. Wong KK, Khong PL, Lin SC, et al. Post-operative magnetic resonance evaluation of children after laparoscopic anorectoplasty for imperforate anus. Int J Colorectal Dis 2005; 20: 33-37.

5. Gangopadhyaya AN, Kumar V, Upadhyaya V, et al. Single-Stage Repair versus Traditional Repair of High Anorectal Malformations, Functional Results' Correlation with Kelly's Score and Postoperative Magnetic Resonance Imaging Findings. Ann Pediatric Surg 2013; 9: 108-113.

6. Hedlund H, Peña A, Rodriguez G, Maza J. Long-term anorectal function in imperforate anus treated by a posterior sagittal anorectoplasty: manometric investigation. J Pediatr Surg 1992; 27: 906-909.

7. Doolin EJ, Black CT, Donaldson JS, et al. Rectal manometry, computed tomography, and functional results of anal atresia surgery. J Pediatr Surg 1993; 28: 195-198.

8. Taccone A, Martucciello G, Fondelli P, et al. CT of anorectal malformation - a postoperative evaluation. Pediatr Radiol 1989; 19: 375-378.

9. Vade A, Reyes H, Wilbur A, et al. The anorectal sphincter after rectal pull-through surgery for anorectal anomalies: MRI evaluation. Pediatr Radiol 1989; 19: 179-183.
10. Gangopadhyay AN, Pandey V, Gupta DK, et al. Assessment and comparison of fecal continence in children following primary posterior sagittal anorectoplasty and abdominoperineal pull through for anorectal anomaly using clinical scoring and MRI. J Pediatr Surg 2016; 51: 430-434.

11. Fukuya T, Honda H, Kubota M, et al. Postoperative MRI evaluation of anorectal malformations with clinical correlation. Pediatr Radiol 1993; 23: 583-586.

12. Kelly JH. Cine radiography in anorectal malformations. J Pediatr Surg 1969; 4: 538-546.

13. Cheu HW, Grosfeld JL. The atonic baggy rectum: a cause of intractable obstipation after imperforate anus repair. J Pediatr Surg 1992; 27: 1071-1073; discussion 1073-1074.

14. Peña A, el Behery M. Megasigmoid: a source of pseudoincontinence in children with repaired anorectal malformations. J Pediatr Surg 1993; 28: 199-203.

15. Jones NM, Humphreys MS, Goodman TR, et al. The value of anal endosonography compared with magnetic resonance imaging following the repair of anorectal malformations. Pediatr Radiol 2003; 33: 183-185.

16. Ichijo C, Kaneyama K, Hayashi Y, et al. Midterm postoperative clinicoradiologic analysis of surgery for high/intermediate-type imperforate anus: prospective comparative study between laparoscopy-assisted and posterior sagittal anorectoplasty. J Pediatr Surg 2008; 43: 158-162. 